\title{
Applications of Momentum-resolved Scanning Transmission Electron Microscopy for Cryo-preserved Radiation Sensitive Materials
}

\author{
Lothar Houben \\ Weizmann Institute of Science, Rehovot, HaMerkaz, Israel
}

Momentum-resolved STEM with multi-segmented and pixelated STEM detectors offers new capabilities for cryo-EM and radiation sensitive materials. Virtual diffraction imaging, phase contrast, differential phase contrast, scanning nanobeam diffraction, ptychography and are more commonly applied in materials science [1] but also provide new tools for the investigation of biological samples.

We have exploited momentum-resolved STEM techniques for radiation sensitive biological and inorganic specimen with cryo-techniques, taking benefit of the EMPAD hybrid pixel detector [2] for momentum resolution experiments and an El-Mul Opal segmented solid state diode detector [3] for better spatial resolution on the expense of momentum sampling. Both detectors are characterized by high sensitivity and single electron counting capabilities that are beneficial for a dose-efficient simultaneous recording of spatial and momentum information, which is in particular relevant for fully hydrated cryo-samples where contrast optimization at low dose is of primary importance.

Momentum-resolved STEM and segmented detection offer new capabilities for tomography, nanodiffraction, phase and orientation mapping under cryo-conditions. Segmented or virtual detector recording aids in mass density segmentation of components such as organelles or inorganic deposits in cryo-tomography data from tilt series, thus adding to the 3D modalities for ultrastructural imaging in cells. For tomography of thick samples and large field of view we anticipate an improved depth of focus while maintaining spatial resolution, vice versa, through additional momentum resolution. Facilitated autofocusing schemes will enhance the throughput of automated tilt series recording and tomography. Scanning nanobeam diffraction experiments in cryogenic conditions give access to early stages of order formation or crystal nucleation stages in various systems where transient states of ordering can be frozen out for ex-situ characterisation. Examples are the noncovalent self-assembly of nanoscale organic systems in aqueous environment or the nucleation and growth of nanomaterials in their native state frozen from solution during wet-chemical synthesis and the influence that ligands have on the mechanism on crystal formation. Nanobeam diffraction in combination with orientational mapping aids in deciphering the intricate construction of mineral structures produced by intracellular biomineralization processes such as the calcification in marine phytoplankton.

\section{References}

[1] C. Ophus (2019). Four-Dimensional Scanning Transmission Electron Microscopy (4D-STEM): From Scanning Nanodiffraction to Ptychography and Beyond. Microsc Microanal 25(3), 1-20.

[2] M.W. Tate et al. (2016). High dynamic range pixel array detector for scanning transmission electron microscopy. Microsc Microanal 22(1), 237-249.

[3] El-Mul Opal STEM detector. https://www.el-mul.com/opal-stem-detector/ 